\title{
Seasonal Variation of Mineral Content of Grazing Grasses in Oil- Producing Area - Ruikpokwu in Rivers State
}

\author{
Asira, Enim Enim \\ Department Of Chemistry, College Of Education, \\ Akamkpa - Cross River State, Nigeria
}

\section{Doi:10.5901/ajis.2013.v2n13p129}

\begin{abstract}
The mineral content of grazing grasses from an oil-producing area (Rukpokwu) in Rivers State, Nigeria was determined from January to June, 2008, to evaluate the effects of seasonal variation of mineral concentrations of grazing grasses, using Izzi in Ebonyi State as control. The grasses analyzed were guinea grass (Pannicum Maximum) and giant star grass (Cynodon nleufuensis). The results obtained indicated high concentrations $(\mathrm{mgl} \mathrm{kg})$ of Potassium $(17.0 \pm 0.2 / 15.0 \pm 0.01)$,

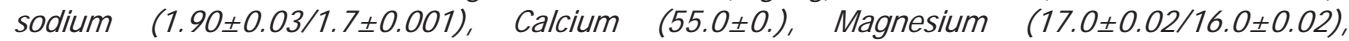
Manganese (5.80 $\pm 0.00 / 5.60 \pm 0.00)$ and Copper (1.30 $\pm 0.01 / 0.90 \pm 0.01)$ and low concentrations of Iron (46.0) and Zinc (3.4 \pm 0.02$)$ for guinea and giant star grasses respectively. During dry season, the concentrations of Calcium (52.0 $\pm 0.2 / 43.0 \pm 0.00)$, Iron (31.0 $\pm 0.01 / 28.0 \pm 0.02)$, Copper $(0.4 \pm 0.01)$ and Zinc $(3.0 \pm 0.02 / 3.1 \pm 0.00)$ in guinea and giant star grasses respectively were observed. In spite of the variation in mineral content of both grasses with season, the grasses still contain sufficient quality of most elements required by livestock
\end{abstract}

Keywords: Grasses, Season, Oil-producing area, minerals and content.

\section{Introduction}

The 1992 earth summit resolved to save the environment and maintain the productive capacity in our plant. Improving the management of hazardous substances, waste toxic chemical and maintaining soil fertility and unpolluted water resources were among the urgent undertakings (United Nations, 1992). Environmental health can be regard as a global public goods and the upkeep of these goods required international cooperation

Grasses are pasture grown to meet the nutritive requirements for optimal lactation and growth of animals reared for subsistence and commercial purpose (Corah and ives, 1981). The grazing grasses, like other plants, depend on the soil, water for the supply of mineral elements, and animal in turn obtain majority of their nutrient from grasses. The primary production of these grasses and their quality in many parts of the world are threatened by contamination of soil and water (Macnairi, 1993; Achangya et al, 1999; Lyangar and Nair, 2000). Even now many livestock are undernourished every day. Vulnerably to toxic waste and contaminated grasses are implicated in poverty both in industrialized and developing world (Lyengar and Nair, 2000 and FAO, 2000). Grazing grasses, being primary producers of food chain, accumulate contaminants from the soil and atmosphere (Harrison and Chingawol, 1989) and contribute mineral elements in harmful concentration to the food chain. The concentrations, of mineral elements in grazing are dependent on soil, plant species, stage of maturity, yield, pasture management and climatic conditions can be important factor in severity of deficiency state by livestock.

The mineral elements commonly found in grazing grasses are classified into micro-minerals, Calcium, Potassium, Sodium, Magnesium, Phosphorus, Nitrogen and Sulphur; and Micro minerals such as Zinc, Iron Copper, Cobalt, Manganese and Selenium. The macro minerals are required in 
substantial amounts in grasses, while the micro minerals are required in small amount (Stanley, 1991).

Rivers State, like other state in southern Nigeria falls within the tropical zone experiences heavy rainfall, high humidity and low temperatures during rainy season and considerable high temperatures low rainfall during dry season (October - March). This variation in the climate affects the concentrations of minerals elements in grazing grasses and soil found in the study area.

The oil-producing area (Rukpokwu) in Rivers State has had her share in the despondent of aquatic essential biota of late. Since 2007, the area has been faced with several petroleum spillages, primarily caused by oil bunker and leakages pipes due to rusts. Also, the areas is one of the homes of oil production in Nigeria.

Some of the grazing grasses found in this giant are elephants grasses (Pannicum purpurum), giant star grass (Cynodon nlemfuensis) and guinea grass (Pannicum maximum)

\section{Aim and Objectives}

The main aim of the study was to determine the effects of seasonal variation on the mineral content of some selected grazing grasses in oil-producing area (Rukpowu) in Rivers State, Nigeria, using Izzi in Ebionyi State as control.

The specific objectives were:

- To determine the total hydrocarbon content of the soil in oil-producing area to ascertain the presence of petroleum oil.

- To determine the mineral content of the soil in the study area.

- To determine the mineral content of the grazing grasses in the said study area

- To determine the variability of the mineral concentrations of the study grasses species with climatic conditions

\section{Scope of the Study}

The scope covers the collection of soil and grasses from the oil- producing area and the control sole every month end from January to June, 2008.

\section{Materials and Methods}

\subsection{Mateials}

\subsubsection{Apparatus}

Polythene bags, shovel, hand forks, evaporating basin, air-evacuation oven, desiccators, weighing balance, knife, $50 \mathrm{ml}$ beakers, pit meter, colorimeter, $200 \mathrm{ml}$ volumetric flask Atomic absorption spectrometer (Perkin Elmer 3II Model) Flame emission photometer, 100ml volumetric flask, stirrer, thermometer and plastic curette $(1 \mathrm{~cm})$

\subsubsection{Reagents}

70-72\% Perchloric acid, 40\% hydrofluoric acid, 95-97\% tetra-oxosulphate VI acid, 6.0m hydro chloric acid, acetic. Acid, 50ml xylene, 40\% tetra-oxochlorate VI, 70\% Trioxonitrate v Acid.

\subsubsection{Experimental grasses}

Giant star grass (Cynodon nlemfuensis) and guinea grass (Pannium maximum) 


\subsection{Methods}

\subsubsection{Collections of soil samples}

Soil samples from oil-producing area (Site A) and non-oil producing area (control) - site B were collected at the end of each month at depths of $0-15 \mathrm{~cm}$ with shovel. The two samples were put in polythene bags labeled OPSS and NOPSS (oil-producing soil samples and non-oil producing soil sample. The samples were bulked, air dried at $85 \mathrm{C}$ for three days filtered and digested (Onyeike and Osiji, 2003).

\subsubsection{Collection of grass samples}

The experimental grass were back at heights of $10 \mathrm{~cm}$ and $5 \mathrm{~cm}$ for guinea and giant star grasses respectively at the end of each month (from January to J une, from three randomly selected area (1. $\mathrm{m}$ in size). These grass species were taken to the laboratory in polythene bags labeled OPGS 1 , 2, 3 and NOPGS 1 . The grasses were then chopped and dried and ground using mortar and pestle and filtered.

\subsubsection{Determination of ph of the siol}

The $\mathrm{pH}$ of the soil samples was determined using $\mathrm{pH}$ Meter (Jenway 3015 model).

\subsubsection{Determination of mineral elements in the soil samples}

Soil phosphorus in the soil was determined by molybdenum method (Onyeike and Osuji, 2003). The concentrations of potassium and sodium ion were determined by flame photometry. Calcium, Copper, Magnesium, Manganese and Zinc ions concentrations were determined by atomic absorption spectrometric method (Perkin Elmer 3110 model). The concentration of iron was done using colorimetric method (Onyeike and Osuji, 2003)

\subsubsection{Determination of mineral content of grass samples}

The concentrations of potassium and sodium were determined by flame emission photometer, iron content was determined by colorimetric method; and Calcium, Magnesium, Manganese, Copper and Zinc were determined by AAS at their respective wavelengths.

\subsubsection{Determination of total hydrocarbon content (THC)}

One gram ( $1 \mathrm{~g}$ ) of soil samples was put into a conical flask and $50 \mathrm{ml}$ of xylene added. The extract was centrifugal for 15 minutes. The absorbance of the extractable hydrocarbon content was obtained using a Hack DR/2010 particle Data logging spectrophotometer at $425 \mathrm{~nm}$. The THC was estimated from a calibrated curve. 


\section{Results}

\subsection{The $\mathrm{pH}$ of the soil samples}

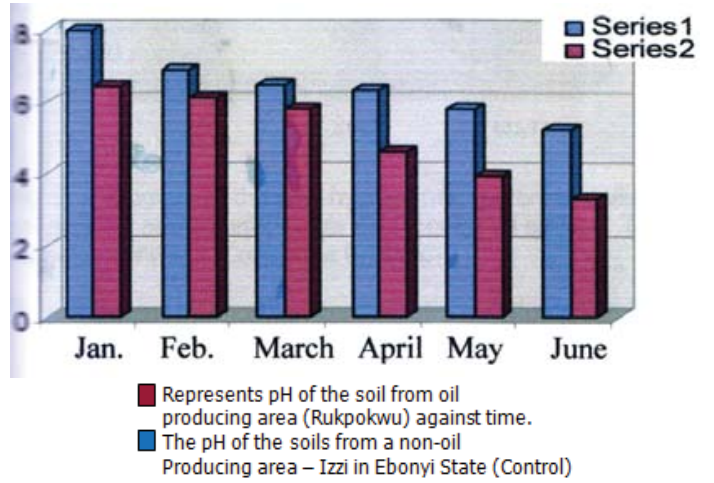

Fig. 1: Histogram of pH of the soil of oil-producing Area (Rukpokwu) and non-oil producing Area, Izzi-Ebonyi State (Control) against time (months)

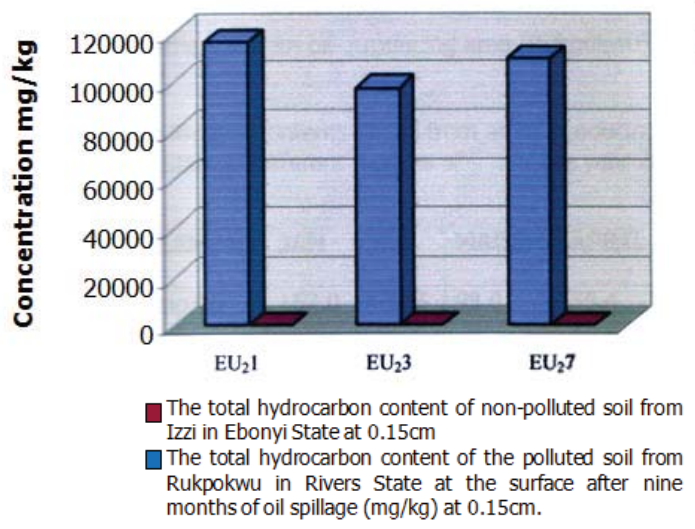

口Series1

口Series2

Fig. 2: Histogram of the total hydrocarbon content of polluted soil and unpolluted soil samples at the surface after nine months soil spillage $(\mathrm{mg} / \mathrm{kg}$ ) from Rukpokwu and Izzi (Control) at $0.15 \mathrm{~cm}$.

Table 1A: Mineral contents of soil from an oil producing area (Rukpokwu) in Rivers State at different months of the year 2008

\begin{tabular}{|l|c|c|c|c|c|c|c|}
\hline Month/Element & Jan & Feb & March & April & May & June & Mean \\
\hline Potassium mg/kg & 152.0 & 133.6 & 98.0 & 55.4 & 103.0 & 127.0 & 111.6 \\
\hline Phosphorus mg/kg & 10.4 & 12.2 & 55.5 & 38.9 & 38.9 & 13.0 & 17.8 \\
\hline Sodium $\mathrm{mg} / \mathrm{kg}$ & 9.0 & 10.0 & 11.6 & 13.5 & 2.6 & 2,9 & 8.27 \\
\hline Calcium $\mathrm{mg} / \mathrm{kg}$ & 716 & 758 & 911.5 & 799.5 & 760.5 & 740.5 & 781.0 \\
\hline Iron $\mathrm{mg} / \mathrm{kg}$ & 350.4 & 346.7 & 30.60 & 30.6 & 301.7 & 301.7 & 309.2 \\
\hline Magnesium mg/kg & 266.5 & 141.0 & 153.0 & 302.0 & 165.1 & 133.0 & 179.7 \\
\hline Manganese mg/kg & 533 & 287.5 & 584 & 604 & 610 & 560.0 & 577.7 \\
\hline Zinc $\mathrm{mg} / \mathrm{kg}$ & 12.6 & 15.0 & 65.5 & 74.4 & 35.0 & 32.7 & 39.2 \\
\hline Copper mg/kg & 6.7 & 8.1 & 9.0 & 7.4 & 7.3 & 7.1 & 7.6 \\
\hline
\end{tabular}


Table 1B: Mineral content of soil from non-oil producing area (Izzi) in Ebonyi State at different months of the 2008.

\begin{tabular}{|l|c|c|c|c|c|c|c|}
\hline Month/Element & Jan & Feb & March & April & May & June & Mean \\
\hline Potassium mg/kg & 201.1 & 227.3 & 130.0 & 100.0 & 136.0 & 210.0 & 167.4 \\
\hline Phosphorus mg/kg & 10.8 & 13.0 & 60.5 & 40.0 & 20.0 & 13.6 & 28.9 \\
\hline Sodium $\mathrm{mg} / \mathrm{kg}$ & 8.9 & 10.7 & 15.0 & 14.8 & 13.2 & 12.0 & 12.2 \\
\hline Calcium mg/kg & 1432 & 1512 & 1623 & 1599 & 1521 & 1481 & 1561.3 \\
\hline Iron $\mathrm{mg} / \mathrm{kg}$ & 300.4 & 296.7 & 256.0 & 256.0 & 251.0 & 251.0 & 269.9 \\
\hline Magnesium mg/kg & 111 & 141 & 153 & 177 & 230 & 266 & 179.7 \\
\hline Manganese mg/kg & 9.6 & 12.0 & 62.6 & 70.0 & 31.5 & 28.7 & 35.77 \\
\hline Zinc mg/kg & 4.7 & 7.2 & 5.5 & 4.8 & 4.3 & 4.6 & 5.18 \\
\hline Copper mg/kg & 210 & 236 & 230 & 288 & 295 & 315 & 276.3 \\
\hline
\end{tabular}

Table 2A: Mineral content of guinea grass from oil producing area (Rukpokwu) of Rivers State (Bracket) represents values of mineral contents of guinea grass from non-oil producing area (I zzi) in Ebonyi State - Control

\begin{tabular}{|l|c|c|c|c|c|c|c|c|}
\hline Month/Element & Jan & Feb & March & April & May & June & Mean & CV \\
\hline \multirow{2}{*}{ Potassium mg/kg } & $1.0 \pm 0.02$ & $1.4 \pm 0.00$ & $1.7 \pm 0.04$ & $2.0 \pm 0.1$ & $17 \pm 0.3$ & $14.0 \pm 0.2$ & 6.18 & 47.7 \\
& $(22.00 \pm 0.1)$ & $1.4 \pm 0.04$ & $(1.7 \pm 0.00)$ & $(3.0 \pm 0.01)$ & $(14.0 \pm 0.2)$ & $(13.0 \pm 0.4)$ & $(5.85)$ & $(52.79)$ \\
\hline \multirow{2}{*}{ Phosphorus mg/kg } & $5.0 \pm 0.01$ & $5.0 \pm 0.03$ & $5.0 \pm 0.1$ & $5.0 \pm 0.1$ & $10.00 \pm 0.03$ & $6.0 \pm 0.2$ & 6.00 & 33.3 \\
& $(9.0 \pm 0.3)$ & $(0.7 \pm 0.00)$ & $(4.0 \pm 0.00$ & $(4.0 \pm 0.00)$ & $(10.00 \pm 0.00)$ & $(8.0 \pm 0.2)$ & $(7.17)$ & $(32.14)$ \\
\hline \multirow{2}{*}{ Sodium $\mathrm{mg} / \mathrm{kg}$} & $0.50 \pm 0.02$ & $0.05 \pm 0.02$ & $0.50 \pm 0.03$ & $43.0 \pm 0.01$ & $1.90 \pm 0.003$ & $0.9 \pm 0.09$ & 0.91 & 67.4 \\
& $(0.60 \pm 0.02)$ & $(1.50 \pm 0.05)$ & $(0.50 \pm 0.00)$ & $(52.0 \pm 0.2)$ & $(0.08 \pm 0.00)$ & $(0.40 \pm 0.00)$ & $(0.75$ & $(52.5)$ \\
\hline \multirow{2}{*}{ Calcium $\mathrm{mg} / \mathrm{kg}$} & $49.0 \pm 0.00$ & $52.00 \pm 0.2$ & $48.00 \pm 0.1$ & $43.0 \pm 0.00$ & $55.0 \pm 0.00$ & $39.0 \pm 0.00$ & 47.0 & 10.71 \\
& $(70 \pm 0.2)$ & $(42.0 \pm 0.2)$ & $(50.0 \pm 0.00)$ & $(51.0 \pm 0.00)$ & $(54.0 \pm 0.2)$ & $(43.0 \pm 0.00)$ & $(50.67)$ & $(10.07)$ \\
\hline \multirow{2}{*}{ Iron $\mathrm{mg} / \mathrm{kg}$} & $30.0 \pm 0.2$ & $31.0 \pm 0.1$ & $48.00 \pm 0.1$ & $42.0 \pm 0.2$ & $46.0 \pm 0.00$ & $14.0 \pm 0.2$ & 34.0 & 34.89 \\
& $(39.0 \pm 0.00)$ & $(23.0 \pm 0.3)$ & $(40.0 \pm 0.00)$ & $(35.0 \pm 0.00)$ & $(59.0 \pm 0.02)$ & $(9.0 \pm 0.00)$ & $31.83)$ & $(48.49)$ \\
\hline \multirow{2}{*}{ Magnesium mg/kg } & $7.00 \pm 0.00$ & $7.00 \pm 0.02$ & $5.00 \pm 0.01$ & $5.0 \pm 0.00$ & $15.00 \pm 0.00$ & $17.0 \pm 0.2$ & 9.50 & 59.3 \\
& $(8.0 \pm 0.02)$ & $(5.0 \pm 0.00)$ & $(4.0 \pm 0.02)$ & $(3.0 \pm 0.00)$ & $(14.0 \pm 0.00)$ & $(13.0 \pm 0.2)$ & $(7.83)$ & $(60.2)$ \\
\hline \multirow{2}{*}{ Manganese $\mathrm{mg} / \mathrm{kg}$} & $3.050 \pm 0.01$ & $2.90 \pm 0.09$ & $4.80 \pm 0.00$ & $3.20 \pm 0.02$ & $5.80 \pm 0.02$ & $3.20 \pm 0.01$ & 3.90 & 29.37 \\
& $(3.5 \pm 0.00)$ & $(4.5 \pm 0.04)$ & $(5.0 \pm 0.00)$ & $(5.0 \pm 0.00)$ & $(5.1 \pm 0.00)$ & $(10.0 \pm 0.02)$ & $(5.51)$ & $(41.66)$ \\
\hline \multirow{2}{*}{ Zinc $\mathrm{mg} / \mathrm{kg}$} & $3.10 \pm 0.00$ & $3.0 \pm 0.02$ & $3.01 \pm 0.01$ & $3.20 \pm 0.00$ & $3.40 \pm 0.02$ & $2.10 \pm 0.00$ & 2.98 & 16.08 \\
& $(3.5 \pm 0.18)$ & $(2.5 \pm 0.00)$ & $(3.3 \pm 0.01)$ & $(4.5 \pm 0.02)$ & $(4.5 \pm 0.00)$ & $(2.4 \pm 0.02)$ & $(3.45)$ & $(12.13)$ \\
\hline \multirow{2}{*}{ Copper $\mathrm{mg} / \mathrm{kg}$} & $0.23 \pm 0.01$ & $0.4 \pm 0.00$ & $0.60 \pm 0.02$ & $0.50 \pm 0.02$ & $1.30 \pm 0.01$ & $0.6 \pm 0.01$ & 0.68 & 29.06 \\
& $(0.20 \pm 0.01)$ & $(0.25 \pm 0.00)$ & $(0.25 \pm 0.004)$ & $(0.42 \pm 0.00)$ & $(1.00 \pm 0.00)$ & $(0.60 \pm 0.01)$ & $(0.45)$ & $(59.3)$ \\
\hline
\end{tabular}

Table 2B: Mineral content of Giant star grass from oil producing area (Rukpokwu) of Rivers State (Bracket) represents values of mineral contents of giant star grass from non-oil producing area (Izzi) in Ebonyi State - Control

\begin{tabular}{|l|c|c|c|c|c|c|c|c|}
\hline Month/Element & Jan & Feb & March & April & May & June & Mean & CV \\
\hline \multirow{2}{*}{ Potassium mg/kg } & $0.9 \pm 0.00$ & $1.2 \pm 0.02$ & $1.5 \pm 0.01$ & $1.8 \pm 0.02$ & $15.0 \pm 0.1$ & $12.0 \pm 0.2$ & 5.4 & \\
& $(1.5 \pm 0.00)$ & $(1.2 \pm 0.02)$ & $(1.5 \pm 0.01$ & $(2.0 \pm 0.02)$ & $(12.0 \pm 0.2)$ & $(11.0 \pm 0.1)$ & $(4.87)$ & \\
\hline \multirow{2}{*}{ Phosphorus $\mathrm{mg} / \mathrm{kg}$} & $5.0 \pm 0.1$ & $4.0 \pm 0.00$ & $4.0 \pm 0.2$ & $5.0 \pm 0.01$ & $12.0 \pm 0.2$ & $7.00 \pm 0.00$ & 6.7 & 32.05 \\
& $(8.0 \pm 0.00)$ & $(6.0 \pm 0.1)$ & $(3.5 \pm 0.00)$ & $(4.8 \pm 0.00)$ & $(9.0 \pm 0.1)$ & $(6.0 \pm 0.3)$ & $(6.21)$ & $(32.5)$ \\
\hline \multirow{2}{*}{ Sodium mg/kg } & $0.5 \pm 0.02$ & $0.6 \pm 0.02$ & $0.6 \pm 0.00$ & $1.3 \pm 0.00$ & $1.70 \pm 0.001$ & $0.8 \pm 0.00$ & 0.917 & 40.5 \\
& $(0.6 \pm 0.02)$ & $(1.6 \pm 0.00)$ & $(0.6 \pm 0.01)$ & $(0.7 \pm 0.01)$ & $(0.70 \pm 0.00)$ & $(0.40 \pm 0.01)$ & $(0.76)$ & $(57.5)$ \\
\hline \multirow{2}{*}{ Calcium $\mathrm{mg} / \mathrm{kg}$} & $50.0 \pm 0.08$ & $43.0 \pm 0.2$ & $52.0 \pm 0.00$ & $43.0 \pm 0.00$ & $55.0 \pm 0.00$ & $39.0 \pm 0.00$ & 47.0 & 13.25 \\
& $(68.0 \pm 0.00)$ & $(40.0 \pm 0.2)$ & $(50.0 \pm 0.00)$ & $(51.0 \pm 0.00)$ & $(53.0 \pm 0.1)$ & $(42.0 \pm 0.00)$ & $(50.67)$ & $(26.44)$ \\
\hline \multirow{2}{*}{ Iron $\mathrm{mg} / \mathrm{kg}$} & $27.0 \pm 0.2$ & $28.0 \pm 0.2$ & $47.0 \pm 0.00$ & $42.0 \pm 0.2$ & $46.0 \pm 0.00$ & $14.0 \pm 0.2$ & 34.0 & 38.6 \\
& $(30.0 \pm 0.2)$ & $(20.0 \pm 0.00)$ & $(38.0 \pm 0.02)$ & $(35.0 \pm 0.00)$ & $(59.0 \pm 0.02)$ & $(9.0 \pm 0.00)$ & $(31.83)$ & $(53.5)$ \\
\hline \multirow{2}{*}{ Magnesium $\mathrm{mg} / \mathrm{kg}$} & $8.0 \pm 0.00$ & $8.0 \pm 0.00$ & $6.5 \pm 0.001$ & $5.0 \pm 0.00$ & $14.0 \pm 0.2$ & $16.0 \pm 0.2$ & 9.58 & 45.9 \\
& $(10.0 \pm 0.2)$ & $(7.0 \pm 0.00)$ & $(5.0 \pm 0.1)$ & $(4.0 \pm 0.00)$ & $(9.2 \pm 0.00)$ & $(8.0 \pm 0.1)$ & $(7.17)$ & $(71.7)$ \\
\hline \multirow{2}{*}{ Manganese $\mathrm{mg} / \mathrm{kg}$} & $3.4 \pm 0.00$ & $2.7 \pm 0.01$ & $4.6 \pm 0.00$ & $3.0 \pm 0.01$ & $5.6 \pm 0.00$ & $3.1 \pm 0.00$ & 3.73 & 30.6 \\
& $(3.6 \pm 0.01)$ & $(4.6 \pm 0.00)$ & $(5.0 \pm 0.01)$ & $(5.0 \pm 0.00)$ & $(5.2 \pm 0.01)$ & $(1.0 \pm 0.00)$ & $(4.07)$ & $(66.7)$ \\
\hline \multirow{2}{*}{ Zinc $\mathrm{mg} / \mathrm{kg}$} & $3.2 \pm 0.01$ & $3.1 \pm 0.00$ & $3.2 \pm 0.01$ & $3.2 \pm 0.00$ & $3.4 \pm 0.00$ & $2.0 \pm 0.00$ & 3.0 & 22.6 \\
& $(3.1 \pm 0.00)$ & $(2.2 \pm 0.01)$ & $(3.1 \pm 0.00)$ & $(4.4 \pm 0.01)$ & $(4.5 \pm 0.00)$ & $(2.1 \pm 0.00)$ & $(3.23)$ & $(33.4)$ \\
\hline \multirow{2}{*}{ Copper $\mathrm{mg} / \mathrm{kg}$} & $02 . \pm 0.02$ & $0.4 \pm 0.01$ & $0.6 \pm 0.01$ & $0.41 \pm 0.00$ & $0.9 \pm 0.01$ & $0.5 \pm 0.00$ & 0.5 & 48.0 \\
& $(0.36 \pm 0.00)$ & $(0.20 \pm 0.00)$ & $(0.20 \pm 0.02)$ & $(0.04 \pm 0.00)$ & $(0.8 \pm 0.02)$ & $(0.5 \pm 0.00)$ & $(0.41)$ & $(54.64)$ \\
\hline
\end{tabular}


Table 3A: Mean mineral content of guinea grass from oil producing area (Rukpokwu) in Rivers State and NRC (1986) recommended mean values for daily meals (DM) for domestic animals.

\begin{tabular}{|c|c|c|c|}
\hline Element & $\begin{array}{c}\text { Research Mean } \\
\text { Values }\end{array}$ & $\begin{array}{c}\text { Nrc (1976) Recommended } \\
\text { Mean Values }\end{array}$ & Remarks \\
\hline $\begin{array}{c}\text { Potassium } \\
\mathrm{mg} / \mathrm{kg}\end{array}$ & 6.18 & $6.00-8.00$ & Within range \\
\hline $\begin{array}{c}\text { Phosphorus } \\
\mathrm{mg} / \mathrm{kg}\end{array}$ & 6.00 & $18.1-22.05$ & Lower than DM requirement \\
\hline $\begin{array}{l}\text { Sodium } \\
\mathrm{mg} / \mathrm{kg}\end{array}$ & 0.97 & $25.00-50.00$ & $\begin{array}{c}\text { Not sufficient for daily } \\
\text { requirement }\end{array}$ \\
\hline $\begin{array}{l}\text { Calcium } \\
\mathrm{mg} / \mathrm{kg}\end{array}$ & 47.5 & 30.0044 .0 & $\begin{array}{c}\text { Higher than daily } \\
\text { requirement }\end{array}$ \\
\hline $\begin{array}{c}\text { Iron } \\
\mathrm{mg} / \mathrm{kg}\end{array}$ & 35.0 & $3.00-6.00$ CAMPEN (1990) & Grossly higher \\
\hline $\begin{array}{l}\text { Magnesium } \\
\mathrm{mg} / \mathrm{kg}\end{array}$ & 9.50 & $6.00-15.00$ & Within range \\
\hline $\begin{array}{c}\text { Manganese } \\
\mathrm{mg} / \mathrm{kg}\end{array}$ & 0.68 & 0.50 & $\begin{array}{l}\text { Slightly higher than daily } \\
\text { requirement }\end{array}$ \\
\hline $\begin{array}{c}\text { Zinc } \\
\mathrm{mg} / \mathrm{kg}\end{array}$ & 3.90 & $0.60-0.70$ & $\begin{array}{c}\text { Higher than daily } \\
\text { requirement }\end{array}$ \\
\hline $\begin{array}{l}\text { Copper } \\
\mathrm{mg} / \mathrm{kg}\end{array}$ & 2.98 & $1.0-3.0$ & Within range \\
\hline
\end{tabular}

Table 3B: Mean mineral content of giant star grass from oil producing area (Rukpokwu) in Rivers State and NRC (1986) recommended mean values for daily meals (DM) for domestic animals.

\begin{tabular}{|c|c|c|c|}
\hline Element & Research Mean Values & $\begin{array}{c}\text { NRC (1976) Recommended } \\
\text { Mean Values }\end{array}$ & Remarks \\
\hline $\begin{array}{c}\text { Potassium } \\
\mathrm{mg} / \mathrm{kg}\end{array}$ & 5.40 & $6.00-8.00$ & Within range \\
\hline $\begin{array}{c}\text { Phosphorus } \\
\mathrm{mg} / \mathrm{kg}\end{array}$ & 6.17 & $18.1-22.05$ & $\begin{array}{c}\text { Grossly lower than daily } \\
\text { requirement }\end{array}$ \\
\hline $\begin{array}{c}\text { Sodium } \\
\mathrm{mg} / \mathrm{kg}\end{array}$ & 0.92 & $25.00-50.00$ & $\begin{array}{c}\text { Grossly lower than daily } \\
\text { requirement }\end{array}$ \\
\hline $\begin{array}{c}\mathrm{Calcium} \\
\mathrm{mg} / \mathrm{kg}\end{array}$ & 47.0 & 30.0044 .0 & $\begin{array}{c}\text { Higher than daily } \\
\text { requirement }\end{array}$ \\
\hline $\begin{array}{c}\text { Iron } \\
\mathrm{mg} / \mathrm{kg}\end{array}$ & 34.0 & $3.00-6.00$ & Within range \\
\hline $\begin{array}{c}\text { Magnesium } \\
\mathrm{mg} / \mathrm{kg}\end{array}$ & 9.58 & $6.00-15.00$ & Within range \\
\hline $\begin{array}{c}\text { Manganes } \\
\mathrm{mg} / \mathrm{kg}\end{array}$ & 0.50 & 0.50 & Within range \\
\hline $\begin{array}{c}\text { Zinc } \\
\mathrm{mg} / \mathrm{kg}\end{array}$ & 3.73 & $0.60-0.70$ & Within range \\
\hline $\begin{array}{c}\text { Copper } \\
\mathrm{mg} / \mathrm{kg}\end{array}$ & 3.00 & $1.00-3.0$ & Within range \\
\hline
\end{tabular}

\section{Discussion}

The $\mathrm{pH}$ of the soil from oil-producing area $(R u k p o k w u)$ in Rivers State declines gradually from dry season to rainy season with peak of 6.4 value recorded in January (see fig.1). Very high hydrocarbon content (most saturated type) was observed in the soil from oil-producing area ( Rukpokwu) (fig. 2) thus confirming the presence of petroleum oil in the soil (see fig. 2). 
The concentrations of potassium (152.0 mg/kg), sodium $(11.6 \mathrm{mg} / \mathrm{kg})$, I ron $350.4 \mathrm{mg} / \mathrm{kg}$ ) and Zinc $(65.5 \mathrm{mg} / \mathrm{kg})$ in the soils from oil producing area recorded during dry season were lower. During rainy season, the concentrations of magnesium $(302 \mathrm{mg} / \mathrm{kg})$, manganese $(604.0 \mathrm{mg} / \mathrm{kg})$ and copper $(7.4 \mathrm{mg} / \mathrm{kg})$ in same soil were higher. Cozzarelli et al (1987) reported elevated concentrations of magnesium and manganese twice their background level at Creasote dump in Pensacote, Florida during rainy season (see tables $1 \mathrm{~A}$ and $1 \mathrm{~B}$ ).

The concentration of potassium in both guinea and giant grasses from the oil producing area was observed to be higher than dry season. Particularly in May $(0.9 \mathrm{mg} / \mathrm{kg})$. This concentration of sodium in both grasses was found to be lower than the total daily meal (DM) ration of $2.50 \mathrm{mg} / \mathrm{kg}$ and $50.0 \mathrm{mg} / \mathrm{kg}$ for growing cattle and lactating cows respectively. NRC, (1976) (see tables 2A \& B).

There was low concentration of phosphorus in both grass species from the oil-producing area throughout the dry season. Gomide (1978) associated the low phosphorus content in young tissues to their being mobile and thus easily translocated from the old tissue to young tissues. During rainy season, high concentration of phosphorus was recorded, particularly in May, $12.0 \mathrm{mg} / \mathrm{kg}$ and $10.0 \mathrm{mg} / \mathrm{kg}$ for guinea and giant star grasses. The mean phosphorus content $6.0 \mathrm{mg} / \mathrm{kg}$ and $6.17 \mathrm{mg} / \mathrm{kg}$ in guinea and giant star grasses respectively from oil-producing area was lower than the requirement for growing and finishing cattle, NRC, (1976).

The concentration of Sodium in both grasses was high during the rainy season. The mean Sodium content in both experimental grasses in oil producing area was lower than the sodium content of $13.0 \mathrm{mg} / \mathrm{kg}$ in the total DM ration for lactating cows NRC, (1976).

High Calcium content of both grasses from oil-producing area was recorded in May (rainy season). However, significant difference in month to month was observed in giant star grass $(P<0.05)$. Nevertheless, a mean Calcium content of $47.0 \mathrm{mg} / \mathrm{kg}$ of the grass species from oilproducing area was found to be higher than (NRC, (1976) Calcium requirement of $30.0 \mathrm{mg} / \mathrm{kg}$ and $44.0 \mathrm{mg} / \mathrm{kg}$ for growing and finishing cattle (see table $3 \mathrm{~A} \& B$ ). This research data confirmed those of Gleadening et al (1983), who reported $57.0 \mathrm{mg} / \mathrm{kg}$ and $41.0 \mathrm{mg} / \mathrm{kg}$ calcium content of some grazing grasses in Arkansas.

The Magnesium content of both grasses in the research area (Rukpokwu) was high during rainy season. This increase in concentration agrees with similar observation recorded my Segel, (1987), who reported elevated concentration of magnesium in grasses in the oil dump in Minnesota in U.S.A during rainy season. Also, significant difference in magnesium content $(P<0.05)$ in months was observed. The low magnesium concentration of the grass samples during dry season could be due to high calcium/magnesium ratio. Stanley (1991). The mean magnesium content $(9.50 \mathrm{mg} / \mathrm{kg})$ and $9.58 \mathrm{mg} / \mathrm{kg}$ for guinea and giant star grasses respectively fails within mean magnesium requirement of $6.0 \mathrm{mg} / \mathrm{kg}$ and $15.0 \mathrm{mg} / \mathrm{kg}$ for growing cattle. (NRC; 1976).

Gradual increase in the concentration of Iron was observed in both grass samples from the research area during the dry season. But during rainy season, decrease Iron concentration was observed (see table $2 \mathrm{~A}$ and $\mathrm{B}$ ). Similar declining iron content with plant maturity had been reported in bromo grass (Brom spp.) Loper and Smith, (1961), during rainy season. Month to month significant difference $(P<0.05)$ was observed in giant star grass I ron content. Cattle through require Iron, the minimum has not been established. (NRC, 1976), Van Campen (1991) gave Iron requirements of $3.0 \mathrm{mg} / \mathrm{kg}$ for calves and $0.5 \mathrm{mg} / \mathrm{kg}$ to $0.6 \mathrm{mg} / \mathrm{kg}$ for mature cattle, values far below those found in this research work. Kechgnessner, (1996) reported that Meadow grass's lowest I ron content was higher than grass species. This may be due to decrease $\mathrm{pH}$ caused by organic acid, which results in dissolution of ferric acid. High values of manganese $(5.80 \mathrm{mg} / \mathrm{kg}$ and $5.60 \mathrm{mg} / \mathrm{kg}$ ) for guinea and giant star grasses were recorded during rainy season. There was month to month significant difference $(P<0.05)$ in manganese content in giant star grass, while guinea grass did not show significant difference. The mean manganese concentration $3.73 \mathrm{mg} / \mathrm{kg}$ and $3.90 \mathrm{mg} / \mathrm{kg}$ in giant star and guinea grasses respectively were higher than NRC (1976) recommend values cattle (table.)

Though it has been observed that season has no effect on Zinc content of guinea and giant 
grasses, Zinc content in the both grasses was observed to be high in rainy season. No month to month significant difference $(P<0.05)$ was recorded. The mean Zinc content of $2.98 \mathrm{mg} / \mathrm{kg}$ and $3.0 \mathrm{mg} / \mathrm{kg}$ in guinea and giant star grasses respectively were within NRC (1976) recommended Zinc content of $1.0 \mathrm{mg} / \mathrm{kg}$ to $3.0 \mathrm{mg} / \mathrm{kg}$ for cattle, hence the grasses may contain sufficient Zinc for cattle performance, except when diet with high calcium suppresses Zinc availability.

Copper content in the experimental grass species was higher in rainy season than dry season. There was significance $(P<0.05)$ difference in months, of copper content in guinea grass, whereas non exist in giant star grass. The mean copper content was slightly higher than $0.5 \mathrm{mg} / \mathrm{kg}$ Copper content recommended for most domestic animals (NRC, 1976) Van Camper, (1990) gave a Copper requirement for growing and finishing cattle as $0.4 \mathrm{mg} / \mathrm{kg}$.

\section{Conclusion}

Data from the study reveal that mineral imbalances/or deficiency in grazing grasses for livestock are related to season and soil pollution. High concentration of total hydrocarbon was confirmed in the soil from oil-producing area (Rukpokwu).

The soil equally recorded high concentrations of potassium, phosphorus and copper during dry season and high concentrations of sodium, calcium, magnesium, manganese and zinc in rainy season. Both guinea and giant star grasses recorded high concentrations of I ron during dry season and high concentrations of potassium, phosphorus, sodium, calcium, magnesium, manganese, copper and zinc in rainy season.

The low concentrations of mineral elements during dry season may be due to natural dilution process and the translocation of mineral elements to the root of grasses as they get matured.

In line with NRC, (1978, 1984 and 1985), mineral requirements recommended for cattle and sheep, the two grass species were observed to be adequate in potassium, magnesium, calcium, Iron, Zinc and Copper. On the contrary, low concentrations of phosphorus and sodium were observed in the two grass species in the study.

High mean content of copper, manganese and Iron in the guinea grass from oil-producing area may be as a result of their ability to build up in grass tissue and thus less susceptible to leaching. The mineral elements analysed in the grass species (guinea and giant star grasses) from oil-producing area (Rukpokwu) suggest that season variation affects the mineral concentrations of grazing grasses and livestock many suffer deficiency in phosphorus and sodium when they depend mainly on guinea and giant star grasses dominant in the polluted area of Rukpokwu, particular during dry season. Equally, high mean content of the trace elements. Irons, manganese and copper, may lead to toxicity in animals that depend mainly or guinea grass in the area.

\section{References}

Achangya, S. K; Chakra borty, P; Lahirisi, S; Raymahashey, B.C; Guha, S. (1999). Arsenic poisoning of Ganges Delta. Nature 40:545

Corah, L. P and Ives, S. (1991). The effects of essential trace minerals on reproduction in beef cattle. Vet.clin. N. American food chain. Animal Pract. 7:41-57.

Cozzarelli, M. I.; Baedecker, M.J; and Hoople, J.A. (1987). Creosote products on the aqueous geochemistry of unsuitable constituents in superficial aquifer. Inc, U.S geological survey programmes, toxic waste ground water contaminants, U.S geological survey open file reports. 87109,pp A-15-16.

F. A. O, (2002). World food chain summit. Five years, headquarters. Rome, Italy. 10-13 June, 2002.

Gomide, J. A. (1978). Mineral composition of grasses and tropical Leguminous forages. Latin American symp on minerals. Nutrition research with grazing ruminants. University of Florida. Gainonesvile. Florida pp 32-40.

Harrison, R. M; Chingawol, M.B. (1989). The Assessment of air and soil as contractors of some trace 
metals to vegetable plants use of a filtered air growth cabinet science tot. Environment. 83:13-34. Kiechgessner,M.(1996). Supply and availability of trace elements. World rev. Animals prod. Pp 41-48.

Lopper, G.M; and Smith, D. (1961). Changes in macro-nutrients composition of the herbage of alfalfa, medium rev. clove ladino clover and bromo grass with advance in maturity. Rep. No. 8 wis. Agric expo. Sta $19 p$.

Lyangar, G.V. and Nair, P.P. (2000). Global out work on nutrition and the environment meeting the challenges of the next millennium. Sci. tot. Environ. 249:331-334.

NRC (1978). Nutrient requirements daily cattle. $5^{\text {th }}$ ed. Nutrients Aca. Of Sci. Washington D.C.

NRC, (1985). Nutrient requirements for domestic animals. Nutrient requirements of daily cattle $6^{\text {th }}$ ed. Nutrient Aca of Sci. Washington D.C.

Onyeike, E.N and Osuji, J.O. (2003). Research techniques in biological and chemical sciences. Published in Nigeria by spring field publishers Ltd. 26:363-402.

Siegel, D.I. (1987) Geochemical facies and mineral dissolution. Bemidj Minnesota, Research site. In: U.S. geological survey programme on toxic waste . 87-109 pp. c13-c15.

Stanley, E.M (1991). Environmental chemistry $5^{\text {th }}$ ed; Lewis publishers Inc. 121 south main street, Chelsea, M.Chigan 48118.

Van Campen, D. (1970) Trace elements in farm animals. Progress in Agric. 13:181-184. 
